\title{
The multivariate coefficient of variation \\ for comparing serum protein electrophoresis techniques \\ in External Quality Assessment schemes
}

\author{
Lixin Zhang • Stéphanie Albarède • Gilles Dumont • \\ Christel Van Campenhout · Jean-Claude Libeer · Adelin Albert
}

\author{
A. Albert $(\bowtie) \cdot$ L. Zhang \\ Medical Informatics and Biostatistics \\ School of Public Health \\ University of Liège, 4000 Liège, Belgium \\ e-mail: aalbert@ulg.ac.be \\ S. Albarède · G. Dumont \\ Direction de l'Évaluation des DIspositifs Médicaux (DEDIM) \\ Agence Française de Sécurité Sanitaire des Produits de Santé (AFSSaPS) \\ 93285 Saint-Denis Cedex, France \\ C. Van Campenhout · J. C. Libeer \\ Department of Clinical Biology \\ Scientific Institute of Public Health \\ 1050 Brussels, Belgium
}


Abstract External Quality Assessment (EQA) schemes are national or transnational programmes designed to control the analytical performance of clinical laboratories and to maintain inter-laboratory variability within acceptable limits. In such EQA programmes, participants are usually grouped by the type of assay technique/equipment they use. The coefficient of variation $(C V)$ is a simple tool for comparing the inter-laboratory reproducibility of such techniques: the lower the $C V$, the better the analytical performance. Serum protein electrophoresis, a laboratory test profile consisting of five fractions (albumin, $\alpha_{1}, \alpha_{2}, \beta$ and $\gamma$ globulins) summing up to $100 \%$ of total proteins, can also be assayed in different ways depending on the media or the analytical principle. We propose a multivariate coefficient of variation for comparing the performance of electrophoretic techniques in EQA, thus extending the univariate $C V$ concept. First, the compositional nature of electrophoretic data requires a one-to-one transformation from the 5-dimensional to the 4-dimensional space. Next, robust estimations of the mean and the covariance matrix are needed to avoid the effect of outliers. The new approach is illustrated on electrophoretic datasets from the French and Belgian national EQA programmes.

Keywords Analytical variability - Electrophoretic techniques - EQA schemes - Equipment performance $\cdot$ Reproducibility $\cdot$ Test profiles 


\section{Introduction}

To control the analytical performance of clinical laboratories, External Quality Assessment (EQA) programmes have been implemented worldwide for many years. These programmes, traditionally organized by healthcare authorities or external agencies, have greatly improved the inter-laboratory compatibility of tests results and hence clinical decision making.

It is known that assay techniques and equipments used by the participants are a source of inter-laboratory imprecision and bias. Not only do calibrators, reagents or kits utilized affect the results, but also other parameters such as the temperature can explain additional variability. Despite international harmonization guidelines and recommendations, the fact that laboratories continue to use different assay methods is an important factor to take into account in EQA programmes. Therefore, EQA organizers generally group participants according to the analytical principle or equipment they use for doing the tests. The statistical analysis of the data is then carried out for each "analytical" group separately. A "robust" mean $(M)$ and standard deviation $(S D)$ are calculated, so that for each individual laboratory result ( $x$ ) a distance $d=|x-M| / S D$ can be derived. Laboratories exceeding a certain critical distance $d^{*}$ from their peers in the group will be considered as "poor-performers". As summary statistics, EQA organizers also compute for each group the coefficient of variation $(\mathrm{CV})$, in order to compare the analytical performance of the various techniques.

Serum protein electrophoresis was only recently implemented in EQA schemes, in particular for controlling the detection and characterization of monoclonal immunoglobulins. Electrophoresis separates serum proteins into five fractions, specifically albumin, $\alpha_{1}, \alpha_{2}, \beta$ and $\gamma$ globulins, reported as absolute values $(\mathrm{g} / \mathrm{L})$ or as relative proportions (expressed in \%) of total serum protein. When expressed in percent, the five electrophoretic fractions sum up to $100 \%$ [1]. As for other analytes, the electrophoretic technique is an important factor likely to influence the final results. These techniques essentially depend on the media (agarose gel, cellulose acetate electrophoresis [1]) or on the analytical principle (e.g., the recently developed capillary zone electrophoresis [2-4]). In a recent paper [5], we proposed a robust multivariate method to analyse EQA electrophoresis data. This method supersedes the traditional approach of viewing each fraction separately (as for ordinary tests) by providing a global assessment of each electrophoretic profile.

The present work was specifically oriented towards the generalization of the coefficient of variation as a way to compare the inter-laboratory variability of electrophoretic techniques, as 
done for single laboratory tests. This is achieved in two steps. First, because of the compositional nature of electrophoretic data (their fractions sum up to 100\%), a one-to-one transformation is applied to the fractions to break the linear constraint and keep only four variables for subsequent calculations. Second, a robust mean and covariance matrix is calculated for the 4-dimensional vector of transformed data. Finally, a multivariate "robust" $C V$ is computed by using a Mahalanobis distance between the mean vector and the origin. The comprehensive development is illustrated on serum protein electrophoresis material from the French and Belgian EQA programmes.

\section{Study material}

Serum protein electrophoresis results were obtained from laboratories participating in the 2004 French and Belgian EQA programmes for the same control sample (04G9). It was a liquid specimen of human origin, which contained a monoclonal IgM-lambda paraprotein concentrated to $5 \mathrm{~g} / \mathrm{L}$, emphasizing a narrow peak in the zone of $\gamma$-globulin of the electrophoretic profile. There were 2376 participants (out of 2965 registered laboratories in the field of immuno-pathology) who actually returned their electrophoresis results to the EQA agencies. Among these, 93 (4\%) electrophoretic profiles for which the sum of the five fractions was not equal to $100 \%$ up to rounding errors (say $\pm 0.2 \%$ ) were discarded from the analysis and considered as not satisfactory. For all other electrophoretic profiles, prior to the statistical analysis, the five fractions were multiplied by a common factor $\lambda=100 \% /$ (sum of observedfractions to sum up to exactly $100 \%$.

A total of 28 different assay techniques were reported by the participants according to media/analytical principle, stain colour and reagent. Only techniques including at least $n=20$ participants were considered in this work. There were 13 such techniques corresponding to a total of 2196 participants (see Table 1). The 13 assay techniques were classified in four major categories: agarose gel-acid blue $(n=227,10 \%)$, agarose gel-amido black $(n=1600,73 \%)$, cellulose acetate membrane-Ponceau $S(n=207,9 \%)$ and capillary zone electrophoresis $(n=162,7 \%)$. As seen in Table 1, there were three techniques based on agarose gel electrophoresis staining with acid blue and five with amido black, three techniques supplied with cellulose acetate membrane staining with Ponceau $\mathrm{S}$ and two 
recently developed capillary zone electrophoreses. Most participating laboratories performed protein electrophoresis using agarose gel electrophoresis (amido black).

Table 1. Distribution into four categories of the 13 serum protein electrophoretic techniques reported in the 2004 French and Belgian EQA programmes with at least 20 participants: principle, stain colour and reagent ( $n=2196$ laboratories)

Electrophoretic technique

Agarose gel electrophoresis (Acid blue) $(n=227)$

Helena Titan ${ }^{\circledR}$ Gel SPE-IFE

Helena SAS-MX SP-10

Helena SAS-MX SP-10 $\beta_{1}-\beta_{2}$

Agarose gel electrophoresis (Amido black) $(n=1600)$

Beckman-Coulter Paragon SPE

Helena Titan ${ }^{\circledR}$ Gel Proteins (HR)

Sebia Hydragel ${ }^{\circledR}$ Protein K20

Sebia Hydragel ${ }^{\circledR} \beta_{1}-\beta_{2}$ Hydrasys

Sebia Hydragel ${ }^{\circledR}$ (Hydratest) (HR) Protein Hydrasys

Cellulose acetate electrophoresis (Ponceau $S)(n=207)$

Helena Titan ${ }^{\circledR}$ III Proteins

Biomidi Midifilm \& Midiplaque Proteins

Sebia Sebiagel

Capillary zone electrophoresis $(n=162)$

Beckman-Coulter Paragon CZE ${ }^{\circledR} 2000$ 65

Sebia Capillarys ${ }^{\circledR} \beta_{1}-\beta_{2} / \beta_{1}-\beta_{2}+$ 


\section{Univariate coefficients of variation}

The coefficient of variation $(C V)$ is a widely used measure in EQA, and in laboratory medicine in general, to assess and compare the reproducibility of techniques and equipments. By definition,

$$
C V=S D / M,
$$

where $M$ represents the mean concentration of the analyte in the control sample and $S D$ the inter-laboratory variability. Since the coefficient of variation is usually expressed in percent, Eq. (1) will be multiplied by $100 \%$. Robust estimates of $M$ and $S D$ are generally required, because EQA datasets often entail outliers or blunders which seriously bias their estimation. Traditionally, $M=P_{50}$, the sample median, and $S D=0.74\left(P_{75}-P_{25}\right)$, where $P_{25}$ and $P_{75}$ are the first and third quartiles of the sample of results, respectively [6]. As an example, Table 2 displays the robust estimates of $M$ and $S D$ for each electrophoretic fraction as obtained from the 903 participants using the "Sebia Hydragel ${ }^{\circledR}$ (Hydratest) (HR) Protein Hydrasys" technique. The corresponding values for all assay techniques are given in Appendix 1.

Table 2. Robust estimates of mean, standard deviation and coefficient of variation for each electrophoretic fraction in the group of laboratories using the "Sebia Hydragel ${ }^{\circledR}$ (Hydratest) (HR) Protein Hydrasys" technique ( $n=903$ laboratories)

\begin{tabular}{lcllll}
\hline & \multicolumn{6}{c}{ Electrophoretic fraction $(\%)$} & & \\
\cline { 2 - 6 } & Albumin & $\alpha_{1}$ & $\alpha_{2}$ & $\beta$ & $\gamma$ \\
\hline Median & 63.40 & 2.30 & 9.70 & 8.40 & 16.40 \\
Standard deviation & 3.03 & 0.30 & 0.74 & 0.74 & 1.55 \\
Coefficient of variation (\%) & 4.8 & 12.9 & 7.6 & 8.8 & 9.5 \\
\hline${ }^{a}$ as obtained from Eq. (1) multiplied by $100 \%$ & & &
\end{tabular}

Once reliable estimates of $M$ and $S D$ are obtained, they can be plugged into Eq. (1) yielding a robust estimate of the $C V$ (see Table 2). Univariate $C V$ s for each electrophoretic fraction and each electrophoretic technique are displayed in Table 3. It is seen that the smallest $C V \mathrm{~s}$ are always observed for the albumin fraction (range: $2.0-8.4 \%$ ), while the largest $C V \mathrm{~s}$ are found for the $\alpha_{1}$-globulin fraction (range: $7.4-30.9 \%$ ) except for "Beckman-Coulter Paragon SPE". When focussing on the electrophoretic techniques, the $C V \mathrm{~s}$ of albumin, $\beta$ and $\gamma$ fractions for the "Beckman-Coulter Paragon CZE ${ }^{\circledR} 2000$ " capillary zone electrophoresis were lower than for the other techniques. Moreover, the other capillary zone electrophoresis 
technique, namely "Sebia Capillarys ${ }^{\circledR} \beta_{1}-\beta_{2} / \beta_{1}-\beta_{2}+$ ", showed high precision for the albumin, $\alpha_{2}, \beta$ and $\gamma$ fractions. The lowest $C V \mathrm{~s}$ for the $\alpha_{1}$ and $\alpha_{2}$ globulins were obtained by using "Beckman-Coulter Paragon SPE". The highest $C V \mathrm{~s}$ of albumin and $\alpha_{2}$-globulin were from "Helena SAS-MX SP-10 $\beta_{1}-\beta_{2}$ " agarose gel electrophoresis (acid blue). For $\alpha_{1}$-globulin, the highest value (30.9\%) was observed in "Helena Titan ${ }^{\circledR}$ III Proteins", followed by "Helena $\operatorname{Titan}^{\circledR}$ Gel Proteins (HR)" technique (29.7\%). The techniques of "Helena Titan ${ }^{\circledR}$ Gel SPEIFE" and "Biomidi Midifilm \& Midiplaque Proteins" provided the highest values for the $\beta$ fraction $(17.5 \%)$ and the $\gamma$-fraction $(11.8 \%)$, respectively.

Table 3. Robust univariate coefficient of variation (\%) of each electrophoretic fraction by technique in the French and Belgian EQA programmes (sample 04G9). Electrophoretic techniques are listed by increasing $C V$ of the albumin fraction

\begin{tabular}{|c|c|c|c|c|c|c|}
\hline \multirow[b]{2}{*}{ Electrophoretic technique } & \multirow[b]{2}{*}{$n$} & \multicolumn{5}{|c|}{$C V(\%)^{\mathrm{a}}$ of electrophoretic fraction } \\
\hline & & Albumin & $\alpha_{1}$ & $\alpha_{2}$ & $\beta$ & $\gamma$ \\
\hline Beckman-Coulter Paragon CZE ${ }^{\circledR} 2000$ & 65 & 2.0 & 8.9 & 6.7 & 4.5 & 2.4 \\
\hline Sebia Capillarys ${ }^{\circledR} \beta_{1}-\beta_{2} / \beta_{1}-\beta_{2}+$ & 97 & 2.6 & 16.9 & 6.5 & 7.9 & 3.8 \\
\hline Sebia Hydragel ${ }^{\circledR} \beta_{1}-\beta_{2}$ Hydrasys & 81 & 3.6 & 11.1 & 6.7 & 7.4 & 9.9 \\
\hline Beckman-Coulter Paragon SPE & 41 & 3.9 & 7.4 & 6.5 & 8.3 & 9.2 \\
\hline Sebia Hydragel ${ }^{\circledR}$ (Hydratest) (HR) Protein Hydrasys & 903 & 4.8 & 12.9 & 7.6 & 8.8 & 9.5 \\
\hline Helena Titan ${ }^{\circledR}$ III Proteins & 141 & 4.9 & 30.9 & 13.6 & 8.8 & 8.7 \\
\hline Helena SAS-MX SP-10 & 86 & 5.2 & 19.8 & 11.4 & 10.9 & 8.9 \\
\hline Helena Titan ${ }^{\circledR}$ Gel Proteins (HR) & 88 & 5.3 & 29.7 & 11.0 & 14.8 & 7.8 \\
\hline Sebia Hydragel ${ }^{\circledR}$ Protein K20 & 487 & 5.5 & 15.4 & 8.8 & 9.4 & 11.4 \\
\hline Helena Titan ${ }^{\circledR}$ Gel SPE-IFE & 121 & 5.9 & 23.7 & 12.8 & 17.5 & 9.9 \\
\hline Biomidi Midifilm \& Midiplaque Proteins & 30 & 6.1 & 17.1 & 10.8 & 5.6 & 11.8 \\
\hline Sebia Sebiagel & 36 & 6.4 & 23.5 & 16.9 & 13.6 & 11.6 \\
\hline Helena SAS-MX SP-10 $\beta_{1}-\beta_{2}$ & 20 & 8.4 & 19.2 & 17.3 & 10.7 & 8.2 \\
\hline
\end{tabular}

${ }^{a} C V$ values are derived from Eq. (1) multiplied by $100 \%$

While it is convenient and straightforward to calculate the $C V \mathrm{~s}$ of the five fractions by technique, it would be more appropriate to provide an overall assessment of the various electrophoretic techniques and to rank them in some way. This can be achieved by computing a multivariate $C V$ for each technique, which accounts for the linear constraint of the 5 fractions and for the correlations between them. In addition, the multivariate $C V$ should not be affected by outlying electrophoretic profiles in the dataset. 


\section{The multivariate coefficient of variation}

Let $\boldsymbol{X}^{\prime}=\left(X_{1}, \ldots, X_{5}\right)$ denote the 5-dimensional electrophoretic profile, where the variables $X_{i}(i=1, \ldots, 5)$ stand for the albumin, $\alpha_{1}, \alpha_{2}, \beta$ and $\gamma$ electrophoretic fractions (expressed in $\%$ ), respectively, and satisfy the relation $X_{1}+X_{2}+X_{3}+X_{4}+X_{5}=100 \%$. Denote by $\overline{\boldsymbol{x}}^{\prime}=\left(\bar{x}_{1}, \ldots, \bar{x}_{5}\right)$ the mean and by $\boldsymbol{S}_{x}$ the variance-covariance matrix of vector $\boldsymbol{X}$. A literature search reveals that little attention has been paid to the multivariate coefficient of variation despite its potential importance. In 1960, Reyment [7] proposed a multivariate generalization of the $C V$ by defining the ratio of the $2 p$-root of the determinant of $S_{x}$ (here $p=5)$ to the length of $\overline{\boldsymbol{x}}$, namely $\sqrt{\bar{x}_{1}^{2}+\cdots+\bar{x}_{5}^{2}}$. Van Valen $[8,9]$ however criticized Reyment's approach and suggested another formula, valid in all cases, in which the numerator of Reyment's formula is replaced by the square-root of the sum of the variances (in his terminology, the total variance), namely $\sqrt{S D_{1}^{2}+\cdots+S D_{5}^{2}}$. Van Valen's approach, while facile to compute, ignores the covariance elements of the $\boldsymbol{S}_{x}$ matrix. Following a suggestion made by Voinov and Nikulin [10] in a totally different context, we chose to define the multivariate coefficient of variation by the expression (multiplied by $100 \%$ )

$$
C V_{M}=1 / \sqrt{\overline{\boldsymbol{x}}^{\prime} \boldsymbol{S}_{x}^{-1} \overline{\boldsymbol{x}}}
$$

where the quantity $\overline{\boldsymbol{x}}^{\prime} \boldsymbol{S}_{x}^{-1} \overline{\boldsymbol{x}}$ represents the Mahalanobis distance of the mean $\bar{x}$ to the origin. Eq. (2) is invariant under change of scale and reduces to Eq. (1) when $p=1$. However, it requires that the matrix $\boldsymbol{S}_{x}$ is invertible, which is not the case for electrophoretic profiles. In a previous paper of our group [5], we avoided this problem by working with a 4-dimensional electrophoretic profile, merely by discarding one of the fractions. Unfortunately, if we use this approach, Eq. (2) yields a different $C V_{M}$ value for each fraction deleted. We strived to circumvent this problem by proceeding with a 2-step procedure as described hereafter.

Step 1. Electrophoresis data transform. The fact that the five electrophoretic fractions sum up to $100 \%$ makes the multivariate statistical analysis unreliable [11]. The linear constraint imposed on the five fractions locates these fractions on a so-called simplex space, which is a subset of the real space. It follows that the covariance matrix, based on the five fractions, is singular and not invertible. Therefore, there is a need to transform the constrained data into an unconstrained real space. Among the family of log-ratio transformations, three are worth 
mentioning, the additive log-ratio (ALR) [11], the centered log-ratio (CLR) [12] and the isometric log-ratio (ILR) transformation [13]. In the present work, we decided to opt for the ILR transform which overcomes the problems of asymmetry of the ALR and of collinearity of the CLR transforms [13]. Let $\boldsymbol{Y}^{\prime}=\left(Y_{1}, \ldots, Y_{4}\right)$ denote the 4-dimensional vector obtained by the ILR-transformation of the electrophoretic profile $\boldsymbol{X}^{\prime}=\left(X_{1}, \ldots, X_{5}\right)$. By definition,

$$
Y_{i}=\sqrt{\frac{i}{i+1}} \ln \frac{\sqrt[i]{\prod_{j=1}^{i} X_{j}}}{X_{i+1}}(i=1, \ldots, 4) .
$$

It should be noted that any zero electrophoretic fractions in Eq. (3) are disallowed. As observed in Eq. (3), the fractions are transformed from five percentages to four dimensionless quantities. An illustration of the use of Eq. (3) is given in Appendix 2. It is also to be remarked that the $Y_{i}$ values depend on the order in which the five fractions are included, while there should be no difference for the final results. After transformation, let $\overline{\boldsymbol{y}}^{\prime}=\left(\bar{y}_{1}, \ldots, \bar{y}_{4}\right)$ and $\boldsymbol{S}_{y}$ denote respectively the sample mean and variance-covariance matrix of $\boldsymbol{Y}$. Observe that now $\boldsymbol{S}_{y}$ can be inverted while $\boldsymbol{S}_{x}$ could not.

Step 2. Robust multivariate estimation. As already mentioned, EQA organizers are traditionally facing the problem of outlying results. This also holds for electrophoretic profiles. The classical mean and covariance matrix (even after the transform above) can be markedly affected even by a single "poor-performer", emphasizing the need for robust estimates [5]. There are plenty of robust methods available, but one of the most attractive rests upon the minimum covariance determinant (MCD) concept developed by Rousseeuw [14]. In addition, the MCD method can also be applied for compositional data [15]. This approach shows many advantages: high robustness, fast algorithm (FAST-MCD) and affine equivariant estimators [16]. According to the FAST-MCD algorithm, the MCD approach consists in finding $h$ observations (in this study, $h=0.75 n$ ) whose classical covariance matrix provides the lowest determinant. The initial location estimate is then the average of these $h$ points and the initial scatter estimate is their covariance matrix. Afterwards, the initial covariance matrix is multiplied by a consistency factor to obtain multivariate normality and a correction factor to be unbiased for small sample sizes [17]. The final covariance matrix $\boldsymbol{S}_{y}$ is obtained by using a weighting step to achieve better efficiency (see "covMcd" function in the "robustbase" package of R 2.9.2 [18]). From the matrix $S_{y}$, it is convenient to derive the correlation matrix $\boldsymbol{R}_{y}$ which is easier to interpret for the user. As a reminder, the correlation 
between two variables is equal to the covariance between the two variables divided by the product of their standard deviations.

The MCD estimates will differ according to which fraction is used as the reference basis for the ILR-transformation. However, in our case, this does not matter since the final robust estimate of the multivariate $C V$ will always be the same. We applied the MCD method to each electrophoretic technique to obtain robust estimates of the mean $(\bar{y})$, covariance matrix $\left(\boldsymbol{S}_{y}\right)$ and correlation matrix $\left(\boldsymbol{R}_{y}\right)$. As an illustration, Table 4 gives the results for the "Sebia Hydragel $^{\circledR}$ (Hydratest) (HR) Protein Hydrasys” technique ( $n=903$ laboratories). The MCD estimates were obtained similarly for all other electrophoretic techniques (data not shown). It should be realized that the correlations between the $Y_{i}$ variables are not the correlations between the electrophoretic fractions.

Table 4. Robust multivariate estimates of mean $(\bar{y})$, covariance matrix $\left(\boldsymbol{S}_{y}\right)$ and correlation matrix $\left(\boldsymbol{R}_{y}\right)$ obtained from laboratories using the "Sebia Hydragel ${ }^{\circledR}$ (Hydratest) (HR) Protein Hydrasys" technique ( $n=903$ laboratories)

\begin{tabular}{|c|c|c|c|c|}
\hline & \multicolumn{4}{|c|}{ ILR- transformed variable } \\
\hline & $Y_{1}$ & $Y_{2}$ & $Y_{3}$ & $Y_{4}$ \\
\hline Mean & 2.34 & 0.17 & 0.25 & -0.41 \\
\hline \multicolumn{5}{|c|}{ Covariance matrix/correlation ${ }^{\mathrm{a}}$ matrix } \\
\hline$Y_{1}$ & 0.014 & 0.0022 & 0.00035 & 0.0026 \\
\hline$Y_{2}$ & 0.31 & 0.0035 & 0.00028 & 0.0017 \\
\hline$Y_{3}$ & 0.038 & 0.061 & 0.0060 & 0.00061 \\
\hline$Y_{4}$ & 0.43 & 0.56 & 0.16 & 0.0025 \\
\hline
\end{tabular}

${ }^{\mathrm{a}}$ Correlations between new variables are given in italic values

After getting the MCD mean $\overline{\boldsymbol{y}}$ and covariance matrix $\boldsymbol{S}_{y}$ from the ILR-transformed results, the value of the multivariate coefficient of variation can be directly calculated for each technique by the following equation (multiplied by 100\%)

$$
C V_{M}=1 / \sqrt{\overline{\boldsymbol{y}}^{\prime} \boldsymbol{S}_{y}^{-1} \overline{\boldsymbol{y}}}
$$

An estimation of the standard error $(S E)$ of $C V_{M}$ can be obtained by the bootstrap method [19]. However, this computer intensive method is sensitive to the presence of outlying observations. Therefore, outliers were first removed from the dataset based on the approach recommended in [17] and then the bootstrap was applied to each cleaned dataset. 
Table 5. Robust multivariate $C V$ s for the protein electrophoretic techniques in the French and Belgian EQA programmes (sample 04G9). The values of $C V_{M}$ have been sorted by increasing order of magnitude. The standard error of the $C V_{M}$ is obtained by the bootstrap method for the cleaned datasets (1000 iterations).

\begin{tabular}{|c|c|c|}
\hline Electrophoretic technique & $n$ & Multivariate $C V^{\text {a }} \pm S E(\%)$ \\
\hline Beckman-Coulter Paragon CZE ${ }^{\circledR} 2000$ & 65 & $2.45 \pm 0.21$ \\
\hline Beckman-Coulter Paragon SPE & 41 & $2.54 \pm 0.25$ \\
\hline Sebia Hydragel ${ }^{\circledR}$ (Hydratest) (HR) Protein Hydrasys & 903 & $3.54 \pm 0.10$ \\
\hline Sebia Hydragel ${ }^{\circledR} \beta_{1}-\beta_{2}$ Hydrasys & 81 & $4.14 \pm 0.28$ \\
\hline Sebia Capillarys ${ }^{\circledR} \beta_{1}-\beta_{2} / \beta_{1}-\beta_{2}+$ & $96^{\mathrm{b}}$ & $4.38 \pm 0.28$ \\
\hline Sebia Hydragel ${ }^{\circledR}$ Protein K20 & 487 & $5.01 \pm 0.18$ \\
\hline Helena SAS-MX SP-10 & 86 & $5.29 \pm 0.53$ \\
\hline Biomidi Midifilm \& Midiplaque Proteins & 30 & $6.02 \pm 0.69$ \\
\hline Helena $\operatorname{Titan}^{\circledR}$ Gel SPE-IFE & 121 & $6.06 \pm 0.45$ \\
\hline Helena $\operatorname{Titan}^{\circledR}$ Gel Proteins (HR) & 88 & $6.95 \pm 0.68$ \\
\hline Helena Titan ${ }^{\circledR}$ III Proteins & 141 & $7.23 \pm 0.45$ \\
\hline Sebia Sebiagel & 36 & $7.81 \pm 0.88$ \\
\hline Helena SAS-MX SP-10 $\beta_{1-} \beta_{2}$ & 20 & $7.82 \pm 1.22$ \\
\hline
\end{tabular}

${ }^{a} C V$ values are derived from Eq. (4) multiplied by $100 \%$

${ }^{\mathrm{b}}$ One laboratory with a zero fraction was discarded

The robust multivariate $C V$ was calculated for each electrophoretic technique using Eq. (4) and expressed in percent. Results are displayed in Table 5 by increasing order of magnitude of $C V_{M}$ together with their standard errors. The "Beckman-Coulter Paragon CZE ${ }^{\circledR} 2000$ " capillary zone electrophoresis technique showed the lowest $C V(2.45 \%)$. By contrast, the "Helena SAS-MX SP-10 $\beta_{1}-\beta_{2}$ " agarose gel electrophoresis (acid blue) technique had the highest $C V_{M}(7.82 \%)$ but was only based on 20 participants ( $S E$ of $1.22 \%$ ).

The values of $C V_{M}( \pm S E)$ were also calculated for the four major electrophoretic categories: agarose gel-acid blue (5.9 $\pm 0.29 \%)$, agarose gel-amido black $(4.4 \pm 0.08 \%)$, cellulose acetate membrane-Ponceau $S(7.8 \pm 0.39 \%)$ and capillary zone electrophoresis $(3.7$ $\pm 0.18 \%)$. Note that the fully "automated" capillary zone electrophoretic technique showed the lowest $C V$. 


\section{Discussion}

The systematic implementation of EQA surveys to control the analytical performance of clinical laboratories generates vast amount of data. The statistical analysis of EQA data pursues several goals: first to provide reliable estimates of the mean (i.e., the concentration of the control specimen) and of the standard deviation (i.e., the inter-laboratory variability); next, to unveil laboratories with outlying results (so called, poor-performers) as compared to the other participants, so that corrective actions or even sanctions can be taken; finally, to highlight unsatisfactory analytical techniques, which can be progressively removed from the market and replaced by better ones. In the latter case, the $C V$ is a useful and widely accepted indicator for comparing the performance of assay techniques or instruments.

Traditionally, EQA schemes have been mainly focussing on single tests (e.g., substrates, enzymes, hormones) so that statistical methods (including robust methods) are pretty straightforward to apply and are easily understood by the laboratory medicine community. When it comes to laboratory profiles, like serum protein electrophoresis, things get somewhat more complicated as profile components (electrophoretic fractions) have to be analysed and interpreted globally and not separately.

In a previous paper of our group [5], we proposed a novel statistical method to analyse EQA electrophoresis data and to get reliable estimates of the mean and dispersion (correlation) matrix of electrophoresis results sent by the EQA participants and identify laboratories with out-of-range profiles. The present work purposed to compare the analytical performance of electrophoretic techniques used by laboratories by calculating a multivariate coefficient of variation. The multivariate $C V$ developed in this paper followed a suggestion made by Voinov and Nikulin in a totally different context. It is equal to the inverse of the square root of the Mahalanobis distance of the mean to the origin. This $C V$ has interesting properties. It is interpreted as a univariate $C V$ and expressed in percent: the lower the $C V$, the better the analytical variability. It is invariant under change of scale (e.g., change of units). It reduces to the classical univariate $C V$ if the profile contains only one test. Nonetheless, it suffers from a few drawbacks. It is highly sensitive to outliers, so that a robust approach is required to obtain a reliable estimate. It can not be calculated if the tests of the profile are linearly related (as it is the case for protein electrophoresis) because the covariance matrix can not be inverted. By deleting one fraction of the electrophoretic profile, however, the covariance matrix is no longer singular. Unfortunately, the $C V_{M}$ will be different for each 
fraction removed. To circumvent these shortcomings, we applied a two-step procedure: (1) the ILR-transform of the 5 electrophoretic fractions to derive a 4-dimensional profile, and (2) to use the MCD method for estimating the mean vector and covariance matrix of the new 4dimensional vector. The multivariate $C V$ can then be derived by utilizing the latter estimates. The method has proven to work quite well and experience has shown that a sample of at least 20 laboratories is needed to get reliable robust estimates. For groups with less than 20 participants, the MCD method is shakier and we recommend simply computing classical estimates of the mean and covariance matrix. Results however have to be considered with utmost care.

The multivariate coefficient of variation was illustrated on a database from the joint French and Belgian EQA electrophoretic programmes, thus allowing having a sufficient number of laboratories within each electrophoretic technique group. The novel approach enabled to rank these techniques by increasing inter-laboratory variability, which could hardly have been done on the basis of the univariate $C V \mathrm{~s}$ of the 5 fractions. Multivariate $C V \mathrm{~s}$ ranged between $2.45 \%$ to $7.82 \%$ and given the large sample sizes in some of the groups, significant $C V$ differences could be highlighted between several techniques.

We believe that the method proposed in this work is quite feasible since the ILR-transform is easy to apply and the R programme to calculate the robust MCD estimates is a freeware that can be downloaded. It was remarked that the value of the multivariate $C V$ does not depend on which fraction of the electrophoretic profile was chosen as the reference (here we selected $\gamma$-globulin but any other fraction could have been used). Our method is applicable whenever a multivariate profile is available. In case data are not compositional, the first step can be skipped and the user can straightforwardly proceed with the robust estimation of the mean and covariance matrix.

\section{Acknowledgments}

This study was partly supported by a research grant from the Scientific Institute of Public Health-Louis Pasteur (Federal Public Service of Social Security, Public Health and Environment), Brussels, Belgium. 


\section{Appendix 1}

Table 6 displays the medians and $S D$ s of the electrophoretic profile (control sample 04G9) for all thirteen electrophoretic techniques considered.

Table 6. Robust univariate estimates of the mean and standard deviation (in parentheses) of each electrophoretic fraction for the thirteen techniques from the 2004 French and Belgian EQA programmes (sample 04G9)

\begin{tabular}{|c|c|c|c|c|c|c|}
\hline \multirow[b]{2}{*}{ Electrophoretic technique } & \multicolumn{6}{|c|}{ Electrophoretic fraction $(\%)$} \\
\hline & $n$ & Albumin & $\alpha_{1}$ & $\alpha_{2}$ & $\beta$ & $\gamma$ \\
\hline Helena Titan $^{\circledR}$ Gel SPE-IFE & 121 & $60.3(3.55)$ & $2.5(0.59)$ & $9.8(1.26)$ & $9.3(1.63)$ & $18.6(1.85)$ \\
\hline Helena SAS-MX SP-10 & 86 & $59.7(3.10)$ & $2.9(0.57)$ & $9.1(1.04)$ & $9.3(1.02)$ & $18.8(1.67)$ \\
\hline Helena SAS-MX SP-10 $\beta_{1}-\beta_{2}$ & 20 & $58.1(4.88)$ & $2.6(0.50)$ & $9.4(1.63)$ & $10.2(1.09)$ & $19.9(1.63)$ \\
\hline Beckman-Coulter Paragon SPE & 41 & $61.4(2.37)$ & $3.0(0.22)$ & $9.2(0.60)$ & $8.7(0.73)$ & $18.2(1.67)$ \\
\hline Helena Titan ${ }^{\circledR}$ Gel Proteins (HR) & 88 & $59.0(3.12)$ & $2.5(0.76)$ & $10.1(1.12)$ & $9.8(1.44)$ & $18.9(1.47)$ \\
\hline Sebia Hydragel ${ }^{\circledR}$ Protein K20 & 487 & $62.3(3.40)$ & $2.4(0.37)$ & $9.2(0.81)$ & $9.4(0.88)$ & $16.6(1.89)$ \\
\hline Sebia Hydragel $^{\circledR} \beta_{1}-\beta_{2}$ Hydrasys & 81 & $63.4(2.29)$ & $2.0(0.22)$ & $8.9(0.59)$ & $9.0(0.67)$ & $16.4(1.63)$ \\
\hline $\begin{array}{l}\text { Sebia Hydragel }{ }^{\circledR} \text { (Hydratest) (HR) } \\
\text { Protein Hydrasys }\end{array}$ & 903 & 63.4( & $2.3(0.30)$ & 4) & $8.4(0.74)$ & 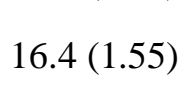 \\
\hline Helena Titan ${ }^{\circledR}$ III Proteins & 141 & 57.9( & 2.4 & 3) & 10.1 & 19.6 \\
\hline $\begin{array}{l}\text { Biomidi Midifilm \& Midiplaque } \\
\text { Proteins }\end{array}$ & 30 & 61.0 & $2.6(0.44)$ & $7.3(0.80)$ & $8.6(0.48)$ & 19.6 \\
\hline Sebia Sebiagel & 36 & $62.6(3.98)$ & $2.6(0.61)$ & $7.7(1.29)$ & $9.0(1.22)$ & $18.6(2.15)$ \\
\hline $\begin{array}{l}\text { Beckman-Coulter Paragon } \\
\text { CZE }^{\circledR} 2000\end{array}$ & 65 & $56.7(1.15)$ & $5.9(0.53)$ & 9.9 & 38) & 18.9 \\
\hline Sebia Capillarys ${ }^{\circledR} \beta_{1}-\beta_{2} / \beta_{1}-\beta_{2}+$ & 97 & $58.2(1.54)$ & $3.5(0.59)$ & $9.4(0.61)$ & $9.6(0.76)$ & $19.5(0.74)$ \\
\hline
\end{tabular}

\section{Appendix 2}

To illustrate the use of Eq. (3), consider the following electrophoretic profile: $X_{1}=63.1 \%$, $X_{2}=2.4 \%, X_{3}=9.7 \%, X_{4}=8.4 \%$ and $X_{5}=16.4 \%$ (total sum $\left.=100 \%\right)$. Then, the transformed data write successively

$$
\begin{aligned}
& Y_{1}=\sqrt{1 / 2} \ln \left(X_{1} / X_{2}\right)=2.31 \\
& Y_{2}=\sqrt{2 / 3} \ln \left(\sqrt{X_{1} X_{2}} / X_{3}\right)=0.19 \\
& Y_{3}=\sqrt{3 / 4} \ln \left(\sqrt[3]{X_{1} X_{2} X_{3}} / X_{4}\right)=0.26 \\
& Y_{4}=\sqrt{4 / 5} \ln \left(\sqrt[4]{X_{1} X_{2} X_{3} X_{4}} / X_{5}\right)=-0.40 .
\end{aligned}
$$




\section{References}

1. Tietz W (ed) (1976) Fundamentals of clinical chemistry. W.B. Saunders Company, Philadelphia, pp 127-134

2. Petrini C, Alessio MG, Scapellato L, Brambilla S, Franzini C (1999) Clin Chem Lab Med 37:975-980.

doi: 10.1515/CCLM.1999.144

3. Bossuyt X, Lissoir B, Mariën G, Maisin D, Vunckx J, Blanckaert N, Wallemacq P (2003) Clin Chem Lab Med 41:704-710.

doi: 10.1515/CCLM.2003.107

4. Bossuyt X, Schiettekatte G, Bogaerts A, Blankaert N (1998) Clin Chem 44:749-759

5. Zhang L, Van Campenhout C, Devleeschouwer N, Libeer JC, Albert A (2008) Accred Qual Assur 13:149-155

6. Libeer JC (1993) External quality assessment in clinical laboratories. European perspectives: today and tomorrow. Higher Education Doctoral Thesis, Antwerpen

7. Reyment RA (1960) Stockholm Contributions in Geology 7: 1-238

8. Van Valen L (1974) Journal of Theoretical Biology 45:235-247

9. Van Valen L (2005) The statistics of variation. In Hallgrímsson, B. and Hall, B.K. (eds), Variation: a central concept in biology. Burlington: Elsevier, pp. 29-47

10. Voinov VG, Nikulin MS (1996) Unbiased estimators and their applications. Vol. 2, multivariate case. Dordrecht: Kluwer, pp. 68

11. Aitchison J (1982) J R Statist Soc B 44:139-177

12. Aitchison $\mathbf{J}$ (1986) The statistical analysis of compositional data. Monographs on statistics and applied probability. Chapman \& Hall, London

13. Egozcue JJ, Pawlowsky-Glahn V, Mateu-Figueraz G, Barceló-Vidal C (2003) Math Geol 35:279-300

14. Rousseeuw PJ (1985) Multivariate estimation with high breakdown point. In: Grossmann W, Pflug G, Vincze I, Wertz W (eds) Mathematical statistics and applications, vol B. Reidel, Dordrecht, pp 283-297

15. Filzmoser P, Hron K (2008) Mathematical Geosciences 40:233-248

16. Rousseeuw PJ, Van Driessen K (1999) Technometrics 41:212-223. doi: $10.2307 / 1270566$

17. Pison G, Van Aelst S, Willems G (2002) Metrika 55:111-123 
18. R development core team (2009) R: A language and environment for statistical computing. R Foundation for Statistical Computing, Vienna, Austria.

ISBN 3-900051-07-0, URL http://www.R-project.org

19. Efron B, Tibshirani RJ (1993) An introduction to the bootstrap. New York: Chapman and Hall 